\title{
Modelo práctico del transformador de distribución trifásico para análisis de transitorios de baja frecuencia: Identificación de parámetros
}

\author{
Practical model of three-phase distribution transformer for low-frequency \\ transients analysis: Parameter identification
}

\author{
Jorge E. Celis-Montero $^{1} \quad$ Ferley Castro-Aranda $^{1} \quad$ Juan A. Martínez-Velasco $^{2}$
}

Recibido 23 de febrero de 2012, aceptado 13 de agosto de 2012

Received: February 23, 2012 Accepted: August 13, 2012

\begin{abstract}
RESUMEN
Para el análisis de los fenómenos transitorios que se originan durante la operación de los transformadores eléctricos, se pueden emplear los modelos implantados en las herramientas de simulación tipo EMTP. Algunos de estos modelos requieren la especificación de parámetros que son difíciles de obtener a partir de ensayo como la característica de saturación de las piernas y los yugos, debido a la interconexión predeterminada de los devanados, o que pueden ser propiedad de los fabricantes de transformadores como las dimensiones relativas del núcleo. En este artículo se propone un modelo para el transformador trifásico de tres columnas que es útil para el análisis de transitorios de baja frecuencia y cuyos parámetros pueden ser determinados mediante pruebas de laboratorio convencionales. El modelo ha sido implantado en EMTP y validado por comparación de resultados obtenidos por simulación con resultados derivados mediante pruebas de laboratorio.
\end{abstract}

Palabras clave: Transformador trifásico, análisis transitorio, modelo, EMTP, saturación.

\begin{abstract}
In order to analyse transient phenomenon originated during electrical transformers operation, models implemented in simulation tools EMTP-type can be used. Some of these models need the specification of parameters that can be difficult to obtain by testing, such as the saturation characteristic of the legs and the yokes, due to the predetermined interconnection of the windings or might be property of transformer's manufacturers, such as the relative dimensions of the core. This paper proposes a three-phase three-legged transformer model that can be used for analysis of low-frequency transients and whose parameters can be determined using conventional laboratory tests. The model has been implemented in an EMTP-type program and validated by comparing simulation results with real measurements obtained from laboratory tests.
\end{abstract}

Keywords: Three-phase transformer, transient analysis, model, EMTP, saturation.

\section{INTRODUCCIÓN}

El desarrollo de un modelo de transformador trifásico adecuado para el análisis de fenómenos transitorios ha sido un objetivo al que se le ha dedicado un gran esfuerzo durante los últimos años [1-3]. Un modelo de transformador es ampliamente dependiente del rango de frecuencias en donde se ubique el transitorio a analizar [3]. Para el análisis de procesos transitorios originados durante la energización de un transformador o en condiciones de ferroresonancia, los cuales contienen frecuencias en un rango que

\footnotetext{
1 GRALTA-Escuela de Ingeniería Eléctrica y Electrónica. Universidad del Valle. Calle 13 No. 100-00. Cali, Colombia. E-mail: jorge.celis@ correounivalle.edu.co; ferley@ieee.org

2 Departament d'Engyniería Elèctrica. Universitat Politècnica de Catalunya. Av. Diagonal 647, CP 08028. Barcelona, España. E-mail:martinez@ee.upc.edu
} 
va de unos pocos $\mathrm{Hz}$ hasta unos pocos $\mathrm{kHz}$ [3], se debe emplear un modelo adecuado para baja frecuencia en el que se incorporen los efectos de la saturación, así como las pérdidas en el núcleo y los devanados del transformador [1].

Entre los modelos de transformador existentes para el análisis de transitorios de baja frecuencia en los que sea fundamental la representación del núcleo se pueden distinguir al menos dos grupos: los modelos basados en dualidad y los modelos híbridos.

Un modelo basado en dualidad incluye una representación completa del núcleo del transformador al que se le añade la representación de los devanados. El modelo resultante incluye únicamente elementos eléctricos, lo que facilita su implantación [5-8]. Por otra parte, el modelo híbrido integra las características del modelo basado en dualidad con una mejor representación de las inductancias de dispersión, además de que es independiente de la estructura del núcleo $[2,9]$.

La gran desventaja a la hora de emplear cualquiera de estos modelos, es la necesidad de recurrir a ensayos de laboratorio que implican conexiones especiales de los devanados para caracterizar adecuadamente las curvas de saturación de las diversas partes del núcleo [3, 10-11], lo que en muchos casos es difícil o imposible de realizar una vez que el transformador ha sido montado en fábrica.

Es por lo tanto importante desarrollar un modelo de transformador trifásico, para el que la determinación de parámetros se pueda realizar a partir de los resultados obtenidos en ensayos de laboratorio convencionales.

En el presente documento se propone un modelo de transformador basado en un modelo magnético desarrollado previamente en otras publicaciones [7, 12-14], que tiene en cuenta la característica de saturación del núcleo e incluye pérdidas por corrientes parásitas y por histéresis, así como un nuevo procedimiento de estimación de parámetros basado en los resultados que se obtienen en ensayos de laboratorio convencionales. El artículo resume el proceso de implantación en EMTP y presenta la validación del modelo mediante ensayos de energización trifásica.

\section{MODELO DEL TRANSFORMADOR TRIFÁSICO BASADO EN DUALIDAD}

Circuito magnético del transformador trifásico

La obtención del modelo de transformador mediante el principio de dualidad parte de la definición de su circuito magnético, para el que se identifican fuentes de fuerza magneto-motriz (FMM), reluctancias magnéticas y flujos magnéticos.

Cuando en un transformador circula corriente por cualquiera de sus devanados (de primario o secundario), se origina una FMM que da lugar a dos flujos magnéticos: el principal, que fluye dentro del núcleo, y el de dispersión, que envuelve el devanado a través del material amagnético (aire o aceite) [16]. Por otro lado, en condiciones de desequilibrio aparece un flujo homopolar que circula por el tanque del transformador.

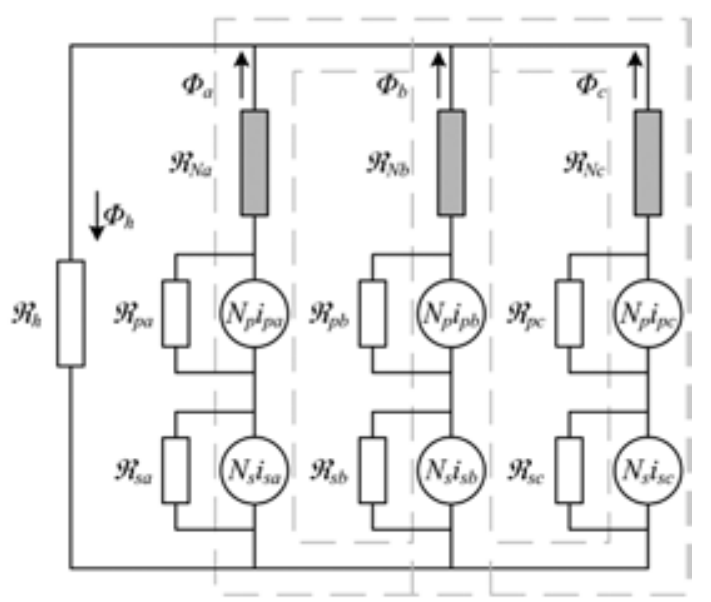

Figura 1. Circuito magnético del transformador trifásico de tres columnas.

La Figura 1 muestra el circuito magnético de un transformador trifásico de tres columnas. Las FMMs que se originan en los devanados a partir de las corrientes primaria y secundaria se representan con las fuentes $N_{p} i_{p a}, N_{p} i_{p b}$ y $N_{p} i_{p c}$ para las corrientes primarias y $N_{s} i_{s a}, N_{s} i_{s b}$ y $N_{s} i_{s c}$, para las corrientes secundarias; las reluctancias lineales $\Re_{p a}, \Re_{p b}, \Re_{p c}$, $\Re_{s a}, \Re_{s b}$ y $\Re_{s c}$ corresponden a los caminos por donde circulan los flujos de dispersión (aire o aceite), mientras que las reluctancias no lineales $\Re_{N a}, \Re_{N b}$ y $\Re_{N c}$ corresponden al núcleo ferromagnético del transformador. Por otra parte, el flujo homopolar $\Phi_{h}$ encuentra un camino de circulación a través de la reluctancia lineal $\Re_{h}$. 
En los modelos propuestos para los transformadores de tres columnas, muchos autores han coincidido en representar el núcleo con varias reluctancias no lineales; de esta manera, se tiene una reluctancia no lineal para cada columna y otras dos que se ubican entre estas últimas para caracterizar los yugos [11], [15]. Sin embargo, este modelo presenta muchas dificultades a la hora de encontrar los parámetros para estos elementos, en especial cuando los devanados están interconectados, puesto que no se pueden efectuar todas las pruebas que se proponen en algunos documentos $[4,15]$.

Esta es la principal razón para desarrollar un modelo más simplificado, cuyos parámetros puedan ser obtenidos mediante ensayos convencionales. Por lo tanto, las reluctancias $\Re_{N a}$ y $\Re_{N c}$ que se muestran en el circuito magnético de la Figura 1 incluyen las reluctancias tanto de la columna como de una porción del yugo.

\section{Obtención del circuito eléctrico equivalente}

Puesto que el análisis de procesos transitorios se suele realizar con circuitos eléctricos, el siguiente paso es la obtención de un circuito equivalente eléctrico del transformador trifásico a partir del modelo magnético anterior, para lo cual se puede aplicar el principio de dualidad [15].

Existen tres reglas generales para aplicar el principio de dualidad: (1) las mallas en el circuito magnético son sustituidas con nodos en el circuito eléctrico y viceversa; (2) las fuentes de FMM se convierten en fuentes de corriente; (3) las reluctancias se convierten en susceptancias [15].

Una vez obtenido el circuito eléctrico equivalente mediante este principio, se deben añadir los elementos que no se representan en el modelo magnético: resistencias de los devanados, pérdidas en el núcleo y acoples capacitivos, así como también transformadores ideales en los terminales del circuito para que los devanados trabajen con tensiones y corrientes reales. En este trabajo no se incluyen los acoples capacitivos entre devanados, puesto que su efecto es poco significativo en el análisis de transitorios de baja frecuencia [1].

Aplicando el principio de dualidad al circuito magnético de la Figura 1 y teniendo en cuenta lo mencionado en el párrafo anterior [4], se puede llegar al circuito eléctrico mostrado en la Figura 2. Las reluctancias no lineales se representan ahora mediante las inductancias no lineales $L_{N a}, L_{N b}$ y $L_{N c}$, a las cuales se han añadido en paralelo las resistencias $R_{m a}, R_{m b}$ y $R_{m c}$, que representan las pérdidas en el material magnético debido a histéresis y corrientes parásitas.

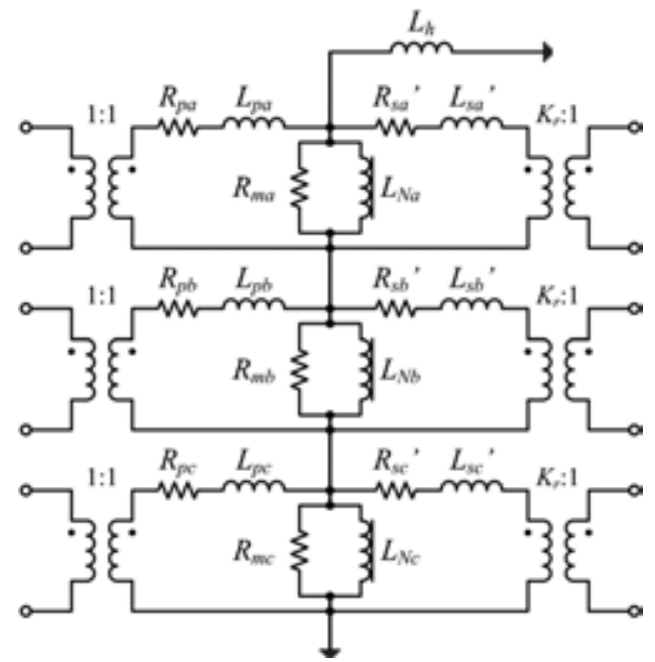

Figura 2. Circuito eléctrico del transformador trifásico de tres columnas.

\section{Identificación de parámetros del modelo}

A partir del circuito eléctrico equivalente obtenido, se deben detallar dos grupos principales de parámetros: los pertinentes a los devanados y los correspondientes al núcleo.

Dentro de los parámetros de los devanados se incluyen la resistencia de cada devanado $\left(R_{p a}, R_{p b}\right.$ y $R_{p c}$ para el lado primario y $R_{s a}, R_{s b}$ ' y $R_{s c}$ ' para el lado secundario), que modela tanto la resistencia eléctrica del material que los conforma como una componente de pérdidas adicionales debidas a los flujos de dispersión que circulan por el material del tanque $u$ otros elementos constructivos del transformador; la inductancia del devanado $\left(L_{p a}\right.$, $L_{p b}$ y $L_{p c}$ para el lado primario y $L_{s a}, L_{s b}$ ' y $L_{s c}$ ' para el lado secundario), que modela los flujos de dispersión alrededor de los devanados, y los transformadores ideales, necesarios para acoplar los parámetros del lado primario y los parámetros del lado secundario.

Por otra parte, los parámetros del núcleo incluyen las resistencias por fase $\left(R_{m a}, R_{m b}, R_{m c}\right)$, que representan 
las pérdidas activas debidas a histéresis y corrientes parásitas en cada columna; las inductancias no lineales por fase $\left(L_{N a}, L_{N b}, L_{N c}\right)$, que modelan la característica de saturación de cada columna del transformador, y la inductancia de secuencia homopolar $\left(L_{h}\right)$, que representa el camino de circulación del flujo homopolar.

Se debe aclarar que existe una asimetría entre los parámetros de cada fase del transformador trifásico de tres columnas debido a su forma constructiva. Aunque es posible suponer iguales los parámetros de los devanados en las tres fases, debido a que su influencia no es muy importante en el análisis de transitorios de baja frecuencia, los parámetros del núcleo no pueden ser iguales en la columna central y en las columnas exteriores si se quiere representar de forma rigurosa la saturación del núcleo ferromagnético del transformador.

\section{Parámetros de los devanados}

Las resistencias y las inductancias de dispersión de los devanados se pueden estimar a partir de los resultados obtenidos en ensayos normalizados de pérdidas bajo carga y de resistencia de devanados [17-19]. Básicamente, se necesitan cuatro parámetros de los ensayos: las pérdidas bajo carga $\left(P_{c c}\right)$, la impedancia porcentual de cortocircuito $\left(Z_{c c}\right)$, la resistencia del devanado primario $\left(R_{\text {prim }}\right)$ y la resistencia del devanado secundario $\left(R_{s e c}\right)$, todos ellos referidos a una misma temperatura.

Es necesario recordar que debido a que los transformadores de distribución tienen generalmente los devanados interconectados, las mediciones de resistencia corresponden a medidas entre terminales. Por consiguiente, se requiere estimar el valor de resistencia por fase para cada tipo de conexión. De este modo, si el devanado está conectado en triángulo (delta), la resistencia por fase es $3 / 2$ de la resistencia medida entre terminales; asimismo, si el devanado está en estrella, la resistencia por fase es $1 / 2$ de la resistencia entre terminales.

Los valores de resistencia y reactancia total vistas desde el lado primario se pueden obtener mediante las expresiones (1) y (2) [20], en las cuales $S_{N o m}$ corresponde a la potencia nominal aparente del transformador y $V_{1 N o m}$ a la tensión nominal del lado primario.

$$
\begin{gathered}
R_{C C}=P_{C C} \cdot \frac{V_{1 N o m}^{2}}{S_{N o m}^{2}} \\
X_{C C}=\left(\frac{V_{1 N o m}}{S_{N o m}}\right)^{2} \cdot \sqrt{\left(\frac{Z_{C C} \cdot S_{N o m}}{100}\right)^{2}-P_{C C}^{2}}
\end{gathered}
$$

La resistencia total $R_{c c}$ en (1) agrupa las resistencias de los devanados más una resistencia que representa la componente de pérdidas activas adicionales [17]. Puesto que no se conoce con exactitud cómo se distribuyen las pérdidas adicionales entre los devanados, se asume que las mismas son del 50\% en el lado primario y de 50\% en el lado secundario.

Se debe recordar que los parámetros de cortocircuito corresponden a parámetros por fase (equivalente en estrella de los devanados), por lo que los parámetros de una conexión en triángulo deben ser llevados a un equivalente en estrella para poder compararlos. Así pues, la resistencia total por fase para cualquier conexión se puede obtener a partir de la siguiente expresión:

$$
R_{C C}=\frac{R_{\text {prim }}+R_{\mathrm{sec}}{ }^{\prime}}{2}+R_{\text {adic }}
$$

La resistencia adicional se divide entre dos:

$$
\frac{R_{\text {adic }}}{2}=\frac{R_{C C}}{2}-\frac{R_{\text {prim }}+R_{\mathrm{sec}}{ }^{\prime}}{4}
$$

$\mathrm{Al}$ añadir esta última expresión a la resistencia medida del devanado, por fase y llevada a un equivalente en estrella, se pueden estimar las resistencias de las ramas primaria y secundaria para el modelo mostrado en la Figura 2:

$$
\begin{aligned}
& R_{P}=\frac{K_{D}}{4} \cdot\left(2 R_{C C}+R_{p r i m}-R_{\mathrm{sec}} \cdot K_{r n}^{2}\right) \\
& R_{S}{ }^{\prime}=\frac{K_{D}}{4} \cdot\left(2 R_{C C}-R_{p r i m}+R_{\mathrm{sec}} \cdot K_{r n}^{2}\right)
\end{aligned}
$$

donde $K_{D}$ es una constante que depende de la conexión del devanado primario, y que es igual a 3 si el devanado está en triángulo y 1 si está en estrella. $K_{r n}$ es la relación entre la tensión nominal primaria y la tensión nominal secundaria del transformador. 
Debido a que se desconoce la distribución de la reactancia de cortocircuito entre los devanados del transformador, y a que no es recomendable asignarla completamente a uno de ellos (ya que en condiciones de carga nula no circula corriente por el secundario y no se producen flujos de dispersión en el mismo), se procede a asumir que la misma se divide en un 50\% entre ambos devanados [1]. Realizando el mismo análisis que en el caso de la resistencia, se puede llegar a la siguiente expresión:

$$
L_{P}=\frac{K_{D}}{2} \cdot \frac{X_{C C}}{\omega}=L_{S}{ }^{\prime}
$$

en donde $\omega$ es la frecuencia angular.

El último parámetro a considerar es la relación de transformación $K_{r}$ del transformador de acople. Esta se calcula de acuerdo al grupo de conexión:

$$
\begin{aligned}
& K_{r}=\sqrt{3} \cdot K_{r n} ; \text { para grupo } \Delta \mathrm{Y} \\
& K_{r}=\frac{1}{\sqrt{3}} \cdot K_{r n} ; \text { para grupo } \mathrm{Y} \Delta
\end{aligned}
$$

\section{Parámetros del núcleo}

Los ensayos que permiten obtener los datos necesarios para estimar los parámetros del núcleo son: el ensayo de magnetización o curva de vacío, el ensayo de pérdidas en vacío [17-18] y el ensayo a secuencia cero [3, 17-19].

Para determinar la característica de magnetización, representada por las inductancias no lineales $\left(L_{\mathrm{Na}}\right.$, $\left.L_{N b}, L_{N c}\right)$ del esquema de la Figura 2, se debe proceder a realizar el ensayo en vacío a varios niveles de tensión, empezando desde cero hasta un porcentaje superior al $110 \%$ de la tensión nominal del lado por el que se alimenta el transformador. Este ensayo se realiza generalmente en el lado de baja tensión del transformador.

Los datos obtenidos (es decir, los valores eficaces de tensión y corriente) son llevados a valores por unidad para su posterior conversión a valores de pico de la corriente de magnetización y del flujo concatenado, empleando para ello la rutina SATURA implantada en el EMTP [21].

Para un transformador trifásico se debe destacar la posibilidad de realizar la prueba de curva de vacío con dos métodos: las tres fases simultáneas con una fuente trifásica (magnetización trifásica) o cada fase individual con una fuente monofásica (magnetización monofásica).

En este trabajo se ha ensayado un transformador trifásico de $2 \mathrm{kVA}-\Delta \mathrm{Y}, 127 / 110 \mathrm{~V}$, para obtener diferentes tipos de curvas de magnetización, aprovechando adicionalmente la posibilidad de acceso a sus terminales, con lo cual se realizaron pruebas desconectando la delta primaria para evaluar su efecto en la saturación. La Figura 3 y la Figura 4 muestran las curvas obtenidas para las fases lateral y central, respectivamente. De las mismas se puede concluir que el devanado en triángulo no tiene un efecto significativo en la magnetización, pues se obtienen curvas muy similares para ambas conexiones.

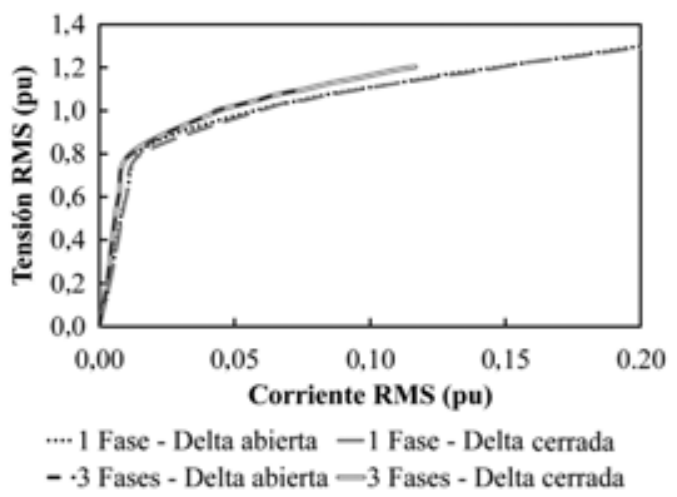

Figura 3. Comparación de la curva de vacío en diferentes condiciones de operación (fases laterales).

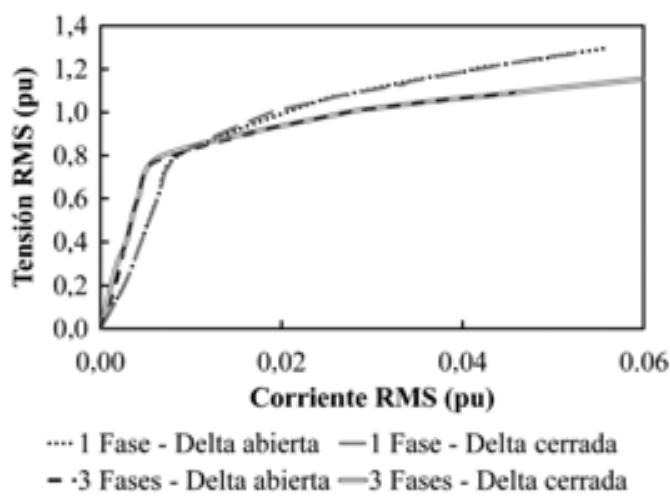

Figura 4. Comparación de la curva de vacío en diferentes condiciones de operación (fase central).

Por otra parte, se observan grandes diferencias en la magnetización trifásica frente a la magnetización monofásica, en especial en la fase central (Fase B). 
Finalmente, se recomienda realizar el ensayo de magnetización monofásica, ya que permite observar el efecto individual de cada columna.

El valor de las pérdidas en vacío $P_{o}$ se obtiene del ensayo correspondiente. Ya se ha mencionado que estas pérdidas son función de la histéresis y las corrientes parásitas en el núcleo ferromagnético; esto implica que también son dependientes de la densidad de flujo magnético y la tensión. Con el fin de reducir la complejidad del modelo en este trabajo se asume que permanecen constantes en todo el rango de tensión. De esta forma, la resistencia de magnetización $R_{m}$ y las pérdidas en vacío $P_{o}$ se relacionan mediante la siguiente expresión:

$$
R_{m}=K_{r n} \cdot \frac{V_{1 N o m}^{2}}{P_{o}}
$$

La resistencia $R_{m}$ así obtenida es un equivalente total del transformador trifásico; sin embargo, se debe discriminar la misma para cada fase debido a la asimetría constructiva del transformador. Para ello se ha realizado un ensayo de magnetización por fase por el lado secundario.

En la Figura 5 se muestra el comportamiento de las pérdidas en vacío para cada fase del transformador. Se puede observar que las fases laterales (Fase A y Fase $C$ ) tienen un comportamiento muy similar, mientras que la fase central (Fase B) presenta menores pérdidas. Evidentemente esto se debe a la asimetría del núcleo, dado que las columnas laterales tienen mayor reluctancia que la central, lo que se traduce en mayores pérdidas activas.

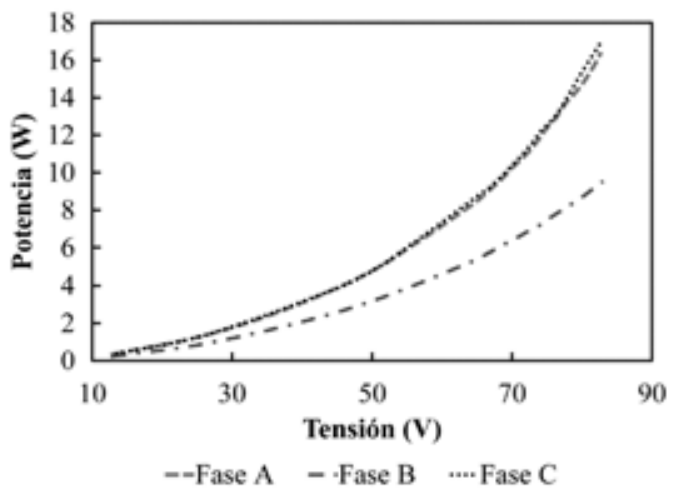

Figura 5. Comportamiento de las pérdidas en vacío por fase.
Ahora se puede determinar el valor de $R_{m}$ para cada fase teniendo en cuenta los datos medidos por el lado secundario. En la Figura 6 se muestra el comportamiento de la resistencia de cada fase. Se puede observar que la resistencia de la columna central (Fase B) es más grande que la de las otras dos.

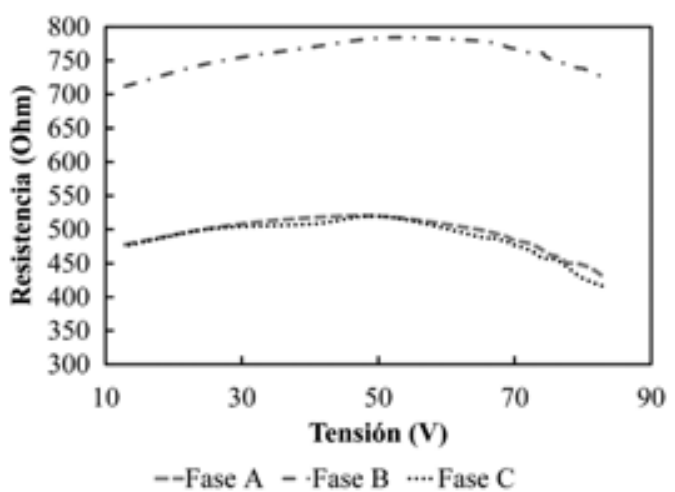

Figura 6. Comportamiento de la resistencia $R_{m}$ por fase.

Por consiguiente, resulta útil estimar una proporción de pérdidas entre cada una de las fases y así poder generalizar el cálculo de las resistencias por fase a partir del valor medido para las pérdidas en vacío. Para ello se ha determinado la distribución porcentual de las pérdidas totales en vacío para cada fase, tal como se muestra en la Figura 7. Del análisis de esta figura, se puede concluir que en la fase central (Fase B) se origina aproximadamente el $25 \%$ de las pérdidas en vacío, mientras que en cada fase lateral (Fase A y Fase C) se origina aproximadamente un $37,5 \%$.

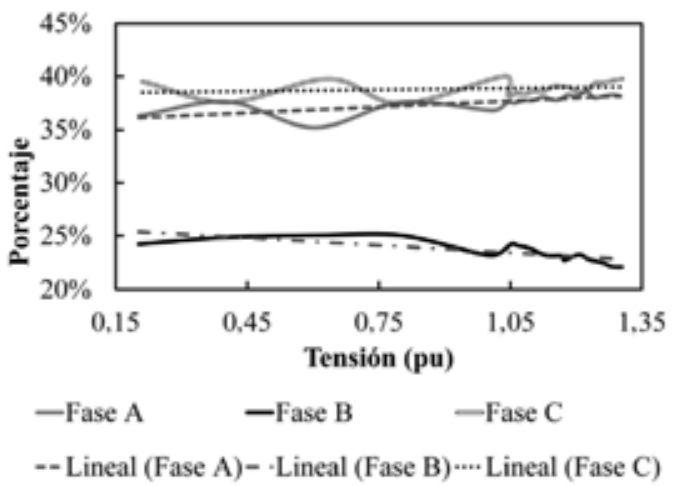

Figura 7. Distribución de las pérdidas de vacío totales entre fases. 
En conclusión, el cálculo de las resistencias de magnetización por cada fase se puede obtener de la siguiente manera:

$$
\begin{gathered}
R_{m a}=R_{m c}=\frac{K_{D}}{3} \cdot \frac{V_{1 N o m}^{2}}{0,375 \cdot P_{o}} \\
R_{m b}=\frac{K_{D}}{3} \cdot \frac{V_{1 N o m}^{2}}{0,25 \cdot P_{o}}
\end{gathered}
$$

El último parámetro a determinar es la inductancia a secuencia homopolar $\left(L_{h}\right)$, la cual se puede estimar mediante el ensayo a secuencia cero que se propone en [3] y [15] para un transformador con devanados interconectados. En dicho ensayo se debe medir la tensión de entrada $\left(V_{h}\right)$, la corriente de entrada $\left(I_{h}\right)$ y las pérdidas activas totales $\left(P_{h}\right)$ por el lado en conexión estrella.

El análisis del circuito de la Figura 8 (para el que se ha considerado devanados en conexión $\Delta \mathrm{Y}$ ) funcionando en régimen permanente permite estimar un valor aproximado de la inductancia a secuencia homopolar. Desde el punto de vista fasorial, se cumple:

$$
K_{r} \cdot \vec{V}_{h}=\vec{V}_{Z S}+\vec{V}_{X h}
$$

Las caídas de tensión en los devanados primario y secundario debido a las corrientes que por ellos circulan, se calculan de acuerdo a las siguientes ecuaciones:

$$
\begin{aligned}
& \overrightarrow{V_{Z S}}=\overrightarrow{I_{L}} \cdot \overrightarrow{Z_{S}} ; \text { donde } \vec{Z}_{S}=R_{S}{ }^{\prime}+j \omega L_{S}{ }^{\prime} \\
& \overrightarrow{V_{X h}}=\overrightarrow{I_{H}} \cdot \overrightarrow{Z_{P}} ; \text { donde } \vec{Z}_{P}=R_{P}+j \omega L_{P}
\end{aligned}
$$

Adicionalmente, la corriente en el devanado secundario se obtiene expresando en forma fasorial la corriente medida durante el ensayo:

$$
\vec{I}_{L}=\frac{1}{3 \cdot K_{r}} \vec{I}_{h}
$$

donde:

$$
\overrightarrow{I_{h}}=\left|I_{h}\right| \angle \phi_{h} ; \phi_{h}=\cos ^{-1}\left(\frac{P_{h}}{V_{h} \cdot I_{h}}\right)
$$

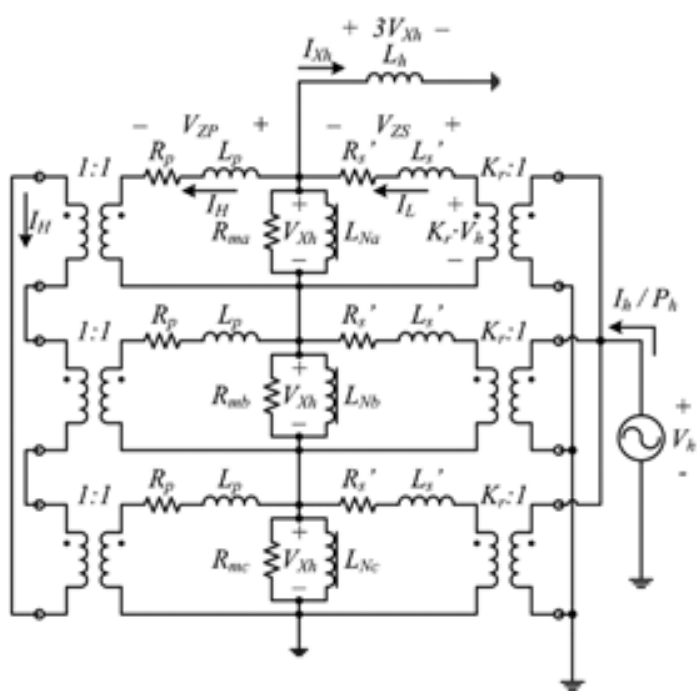

Figura 8. Ensayo para estimar la inductancia de secuencia homopolar.

Por lo que respecta a la corriente en el devanado primario, esta se puede determinar de la siguiente forma:

$$
\overrightarrow{I_{H}}=\frac{K_{r} \cdot \vec{V}_{h}-\vec{I}_{L} \cdot \vec{Z}_{S}}{\vec{Z}_{P}}
$$

Posteriormente se puede determinar la impedancia a secuencia homopolar:

$$
\vec{Z}_{h}=\frac{3 \cdot \overrightarrow{V_{X h}}}{\overrightarrow{I_{X h}}}=\frac{3 \cdot \overrightarrow{I_{H}} \cdot \overrightarrow{Z_{P}}}{\overrightarrow{I_{L}}-\overrightarrow{I_{H}}}=\frac{3 \cdot \vec{Z}_{P}}{\frac{\overrightarrow{I_{L}}}{\overrightarrow{I_{H}}}-1}
$$

Aunque la impedancia a secuencia homopolar tiene una parte resistiva y una parte inductiva (es decir, $\vec{Z}_{h}=R_{h}+j X_{h}$, donde $X_{h}=\omega \cdot L_{h}$ ), en este trabajo el valor de la impedancia se aproxima por el de la reactancia. Posteriormente, se obtiene el valor de la inductancia a secuencia homopolar dividiendo la reactancia $X_{h}$ por la frecuencia de operación del transformador.

\section{IMPLANTACIÓN DEL MODELO EN EMTP}

En esta sección se detalla el proceso de implantación del modelo propuesto en este trabajo en el programa de análisis de transitorios electromagnéticos EMTP. 
En el caso de las resistencias en los devanados $\left(R_{P}, R_{S}^{\prime}\right)$ y las resistencias que representan las pérdidas en el núcleo $\left(R_{m a}, R_{m b}, R_{m c}\right)$, se han utilizado elementos lineales. De igual manera, las inductancias de dispersión de los devanados $\left(L_{P}\right.$, $\left.L_{S}{ }^{\prime}\right)$ y la inductancia a secuencia homopolar $\left(L_{h}\right)$ se han representado con elementos inductivos lineales.

Para representar las ramas de magnetización de cada columna del transformador trifásico se han utilizado las inductancias del tipo 93. Estas inductancias son dependientes de la corriente, y se especifican a partir de la relación entre los valores de pico del flujo concatenado y la corriente de magnetización. Como ya se ha mencionado, esta relación se obtiene mediante la rutina SATURA de EMTP, a partir de los datos obtenidos en ensayos.

En el modelo implantado, adicionalmente se han agregado los transformadores ideales para adecuar los niveles de tensión y corriente por ambos lados del transformador trifásico. Para evitar puntos flotantes, a estos transformadores se les han añadido acoples resistivos a tierra de muy alto valor.

En la Figura 9 se ilustra el esquema final implantado en EMTP, en el que el transformador opera en conexión $\mathrm{Y} \Delta$ con la estrella puesta a tierra. El esquema de la figura incluye el equivalente de red, representado mediante una fuente de tensión ideal en serie con un elemento RLC trifásico, y los interruptores controlados por tiempo para la conexión del transformador a la red.

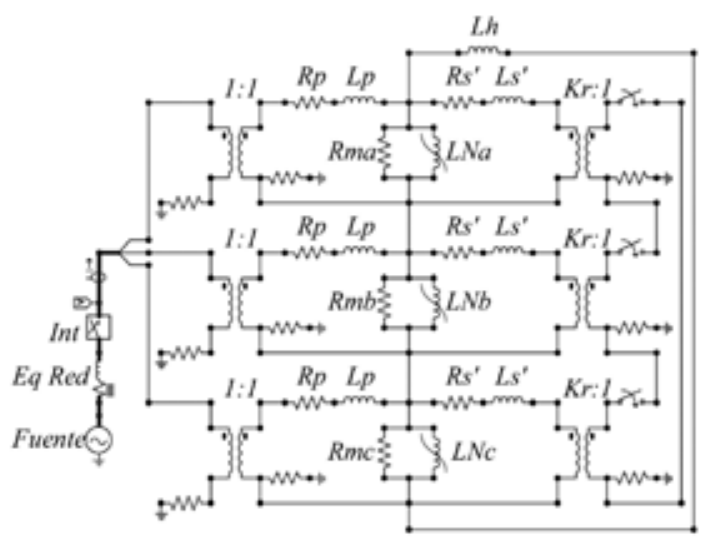

Figura 9. Implantación del modelo de transformador de tres columnas en EMTP.

\section{VALIDACIÓN DEL MODELO}

En esta sección sólo se estudiará un caso de energización trifásica en el que se comparan las corrientes inrush y en régimen permanente.

Para los ensayos se ha empleado un transformador tipo seco y $2 \mathrm{kVA}$ de potencia nominal, con tensiones nominales $110 / 127 \mathrm{~V}$, y grupo de conexión $\mathrm{Y} \Delta$. La Tabla 1 muestra los datos obtenidos con el ensayo a temperatura ambiente. Estos datos han sido procesados de acuerdo a lo descrito previamente, para estimar los parámetros del modelo implantado en EMTP.

Es importante aclarar que durante la ejecución de las pruebas de laboratorio se ha intentado reducir el impacto de aquellas variables que pueden jugar un papel decisivo en los resultados esperados, como es el caso de la magnetización remanente. Es por ello que antes de realizar una prueba de energización se efectúa un procedimiento de desmagnetización general de acuerdo a lo propuesto en [22].

Tabla 1. Datos de ensayo del transformador bajo estudio.

\begin{tabular}{|c|c|}
\hline \multicolumn{2}{|c|}{ Datos de ensayo estándar } \\
\hline$P_{C C}(\mathrm{~W})$ & 89,54 \\
\hline$Z_{C C}(\%)$ & 4,61 \\
\hline$R_{\text {prim }}(\mathrm{m} \Omega)$ & 322,39 \\
\hline$R_{s e c}(\mathrm{~m} \Omega)$ & 271,51 \\
\hline$P_{o}(\mathrm{~W})$ & 15,46 \\
\hline \multicolumn{2}{|c|}{ Datos de ensayo a secuencia homopola } \\
\hline Tensión (V) & 2,00 \\
\hline Corriente (A) & 35,56 \\
\hline Pérdidas (W) & 69,61 \\
\hline
\end{tabular}

Los ensayos de energización trifásica fueron realizados por el lado en estrella aplicando el valor de tensión nominal del devanado. La energización se realizó empleando interruptores de baja tensión, los cuales presentan oscilaciones normales durante su operación. Durante el ensayo se registraron las tensiones entre fase y neutro y las corrientes de línea, tanto al inicio de la energización como una vez que se alcanzó el régimen permanente. 
En el modelo implantado en EMTP se ha buscado representar las condiciones más cercanas posibles a las obtenidas en los ensayos; por ejemplo, un flujo remanente cero en las inductancias no lineales.

La Figura 10 presenta la comparación de las ondas de tensión fase-neutro medidas en el laboratorio y las obtenidas en la simulación. Como se puede comprobar, se ha conseguido establecer el mismo instante de energización para ambos casos.

La Figura 11 muestra las corrientes Inrush obtenidas mediante ensayo y por simulación. Se puede destacar que los resultados obtenidos a partir de simulación están muy próximos a los obtenidos en el laboratorio. La principal diferencia radica en el primer pico de corriente, el cual depende del grado de saturación del núcleo ferromagnético [4], y por tanto del flujo remanente inicial, ya que no fue posible conseguir que el núcleo estuviera completamente desmagnetizado.

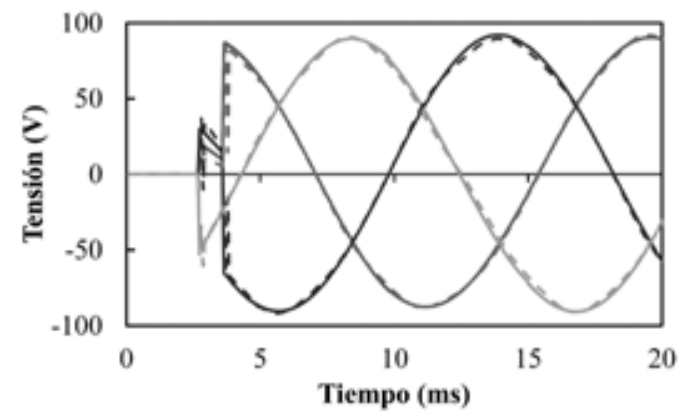

-Fase A (ATP) - Fase B (ATP) -Fase C (ATP)

- -Fase A (Real)- -Fase B (Real) - -Fase C (Real)

Figura 10. Punto de energización.

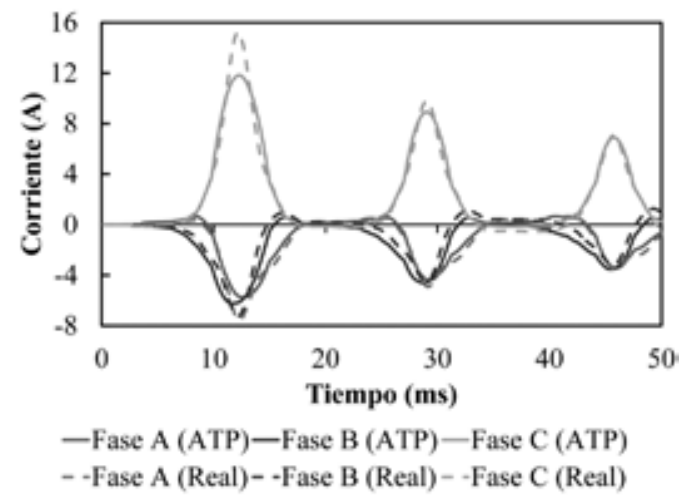

Figura 11. Comparación de corrientes Inrush.

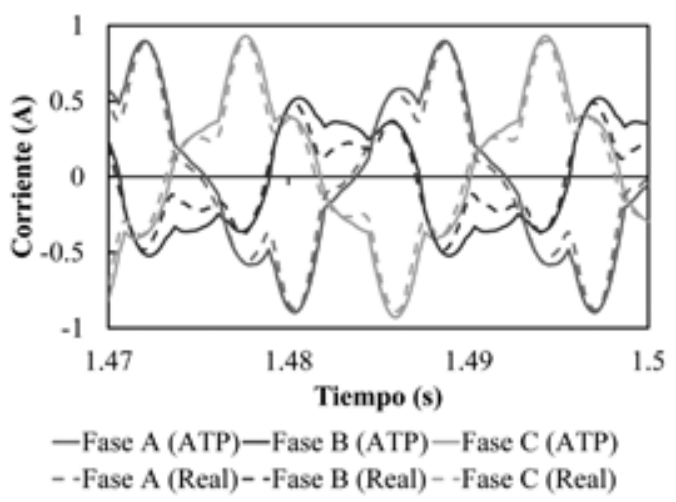

Figura 12. Comparación de corrientes en régimen permanente.

Finalmente, en la Figura 12 se comparan las ondas de corriente en régimen permanente obtenidas mediante simulación respecto a las registradas en el laboratorio, encontrándose gran similitud entre los valores pico y las formas de onda para cada una de las fases.

Los resultados obtenidos demuestran la pertinencia del modelo propuesto. Además de las razones ya mencionadas, se puede añadir que las diferencias entre resultados obtenidos mediante ensayo y simulación se deben también al hecho de no incluir el fenómeno de histéresis en la característica de saturación del núcleo y considerar constantes las pérdidas en el núcleo.

\section{CONCLUSIONES}

En este artículo se ha presentado un modelo de transformador trifásico de tres columnas basado en el principio de dualidad y su implantación en EMTP. El modelo es adecuado para el análisis de transitorios de baja frecuencia. Los resultados obtenidos muestran su aplicabilidad y fiabilidad.

Aunque el modelo magnético utilizado ya ha sido propuesto en otras publicaciones, en este trabajo se ha hecho un esfuerzo en sintetizar el uso del modelo y en la determinación de los parámetros del mismo a partir de los resultados obtenidos en ensayos convencionales, lo cual facilita su aplicación.

La ventaja del modelo radica en representar individualmente cada columna del núcleo mediante su propia curva de saturación y su propia componente de 
pérdidas activas, de manera que se logra caracterizar adecuadamente la asimetría magnética propia del núcleo de tres columnas.

El modelo empleado permite una fácil extensión a otros tipos constructivos de transformador; por ejemplo, el transformador trifásico de cinco columnas.

\section{REFERENCIAS}

[1] J.A. Martinez-Velasco and B. Mork, "Transformer Modeling for Low-and Mid-Frequency Transients-A Review". IEEE Transactions on Power Delivery. Vol. 20, Issue 2, pp. 1625-1632. April, 2005. ISSN: 0885-8977. DOI: 10.1109/ TPWRD.2004.833884.

[2] B. Mork, F. Gonzalez, D. Ishchenko, D.L. Stuehm and J. Mitra. "Hybrid Transformer Model for Transient Simulation-Part I: Development and Parameters". IEEE Transactions on Power Delivery. Vol. 22, Issue 1, pp. 248-255. June, 2007. ISSN: 1932-5517. DOI: 10.1109/PES.2007.385620.

[3] F. de León, P. Gómez, J.A. Martinez-Velasco and M. Rioual. "Transformers". Chapter 4 of Power System Transients. Parameter Determination. J.A. Martinez-Velasco (ed.). CRC Press, p. 644. Boca Raton, USA. 2009. ISBN: 978-1-4200-6529-9.

[4] N. Chiesa. "Power Transformer Modeling for Inrush Current Calculation". PhD Thesis. Norwegian University of Science and Technology. NTNU, p. 238. Trondheim, Norway. 2010. ISBN 978-82-471-2086-6.

[5] X. Chen and S.S. Venkata. "A Three-Phase Three-Winding Core-Type Transformer Model for Low-Frequency Transient Studies". IEEE Transactions on Power Delivery. Vol. 12, Issue 2, pp. 775-782. April, 1997. ISSN: 0885-8977. DOI: 10.1109/61.584369.

[6] B. Mork. "Five-legged Wound-Core Transformer Model: Derivation, Parameters, Implementation and Evaluation". IEEE Transactions on Power Delivery. Vol. 14, Issue 4, pp. 1519-1526. October, 1999. ISSN: 0885-8977. DOI: 10.1109/61.796249.

[7] R. López García. "Desarrollo y Validación de Modelos de Transformadores Monofásicos y Trifásicos con Saturación para el Análisis de Armónicos en Sistemas de Potencia”.
Tesis Doctoral. Universidad Politécnica de Cataluña, p. 288. Barcelona, España. 2000.

[8] F. Zhalefar and M. Sanaye-Pasand. "Transformer Core Modeling as Applied to Slow Transients and Protective Studies". 2006 IEEE Power India Conference, pp. $397-$ 403. June, 2006. ISBN: 0-7803-9525-5. DOI: 10.1109/POWERI.2006.1632544.

[9] H. Hoidalen, B. Mork, F. Gonzalez, D. Ishchenko and N. Chiesa. "Implementation and Verification of the Hybrid Transformer Model in ATPDraw". Electric Power Systems Research. Vol. 79, Issue 3, pp. 454-459. March, 2009. ISSN: 0378-7796. DOI: 10.1016/j.epsr.2008.09.003.

[10] B. Mork, F. Gonzalez, D. Ishchenko, D.L. Stuehm and J. Mitra. "Hybrid Transformer Model for Transient Simulation -Part II: Laboratory Measurements and Benchmarking". IEEE Transactions on Power Delivery. Vol. 22, Issue 1, pp. 256262. June, 2007. ISSN: 1932-5517. DOI: 10.1109/PES.2007.385623.

[11] D. Stuehm. "Three-Phase Transformer Core Modeling”. Final Report, Bonneville Power Administration. Portland (OR), p. 135. USA. 1993.

[12] J. Pedra, L. Sainz, F. Corcoles, R. López and M. Salichs. "PSPICE Computer Model of a Nonlinear Three-Phase Three-Legged Transformer". IEEE Transactions on Power Delivery. Vol. 19, Issue 1, pp. 200-207. January, 2004. ISSN: 0885-8977. DOI: 10.1109/TPWRD.2003.820224.

[13] M.A.S. Masoum and P.S. Moses. "Impact of Balanced and Unbalanced Direct Current Bias on Harmonic Distortion Generated by Asymmetric Three-Phase Three-Leg Transformers". IET Electric Power Applications. Vol. 4, Issue 7, p. 507. February, 2010. ISSN 1751-8660. DOI: 10.1049/iet-epa.2009.0311.

[14] P.S. Moses, M.A.S. Masoum and H.A. Toliyat. "Dynamic Modeling of Three-Phase Asymmetric Power Transformers With Magnetic Hysteresis: No-Load and Inrush Conditions". IEEE Transactions on Energy Conversion. Vol. 25, Issue 4, pp. 1040-1047. December, 2010. ISSN: 0885-8969. DOI: 10.1109/TEC.2010.2065231. 
[15] N. Chiesa. "Power Transformer Modelling: Advanced Core Model". Master Thesis. Politecnico Di Milano, p. 146. Milan, Italy. 2005.

[16] J.J. Winders. "Power Transformers. Principles and Applications". Marcel Dekker, p. 286. NY, USA. 2002. ISBN: 0-8247-0766-4.

[17] IEEE Std. C57.12.90-2010. "IEEE Standard Test Code for Liquid-Immersed Distribution, Power and Regulating Transformers". 2010.

[18] IEEE Std. C57.12.91-2001. "IEEE Standard Test Code for Dry-Type Distribution and Power Transformers". 2001.

[19] IEC Standard 60076-1 Ed 3.0. "Power Transformers-Part 1: General”. 2011.
[20] J.A. Martínez-Velasco y F. de León. "Circuito equivalente de un transformador con regulación". Ingeniare. Revista chilena de ingeniería. Vol. $19 \mathrm{~N}^{\circ} 1$, pp. 93-109. Marzo-abril 2011. ISSN 0718-3305. DOI: 10.4067/S0718-33052011000100010.

[21] H.W. Dommel. "ElectroMagnetic Transients Program (EMTP) Theory Book". Bonneville Power Administration, p. 483. Portland, USA. 1995.

[22] I. Farzadfar. "An Inrush Current Model for Core Type Transformers". Master Thesis. University of Manitoba, p. 142. Winnipeg, Canada. 1997. 